\title{
Plasmon resonances and electron phase shifts near Au nanospheres
}

\author{
T. C. Petersen, ${ }^{1, a)}$ M. Bosman, ${ }^{2}$ V. J. Keast, ${ }^{3}$ and G. R. Anstis ${ }^{4}$ \\ ${ }^{1}$ Australian Key Centre for Microscopy and Microanalysis, The University of Sydney, \\ Sydney, New South Wales 2006, Australia \\ ${ }^{2}$ Institute of Microelectronics, A*STAR (Agency for Science, Technology and Research), \\ 11 Science Park Road, Singapore 117685, Singapore \\ ${ }^{3}$ School of Mathematical and Physical Sciences, The University of Newcastle, \\ Callaghan, New South Wales 2308, Australia \\ ${ }^{4}$ Department of Applied Physics, University of Technology, Sydney, P.O. Box 123, \\ Broadway, Sydney, New South Wales 2007, Australia
}

(Received 10 July 2008; accepted 20 August 2008; published online 10 September 2008)

\begin{abstract}
Quantitative phase maps from $\mathrm{Au}$ and polystyrene nanospheres were obtained using the transport of intensity equation approach for phase retrieval in the electron microscope. These were compared to surface plasmon maps, as generated by electron energy loss spectroscopy. Large phase excursions were observed in the vacuum regions surrounding the Au nanospheres, yet not near adjacent polystyrene nanospheres. It is proposed that these phase excursions are associated with surface plasmon excitation. These results suggest that phase retrieval offers an alternative means for sensing and interpreting the optical response of nanostructured metals. (c) 2008 American Institute of Physics. [DOI: 10.1063/1.2980505]
\end{abstract}

The motivation for the work reported here stemmed from preliminary observations in the transmission electron microscope (TEM) of unusual electron phase shifts induced near metal surfaces. Here we explore the possibility that these observations are related to the excitation of surface plasmons, which can be mapped in a scanning TEM (STEM) combined with electron energy loss spectroscopy (EELS). ${ }^{1,2}$

Electron phase shifts can be detected with off-axis electron holography, using a TEM equipped with an electrostatic biprism. ${ }^{3}$ In this work, we use an alternative method of inline holography based on the transport of intensity equation (TIE). ${ }^{4}$ This approach relates spatial gradients in the time averaged irradiance $I\left(\mathbf{r}_{\perp}, z\right)$ and phase $\phi\left(\mathbf{r}_{\perp}, z\right)$ to the variation in $I\left(\mathbf{r}_{\perp}, z\right)$ along the optic axis $z$,

$$
\nabla_{\perp} \cdot\left\{I\left(\mathbf{r}_{\perp}, z^{\prime}\right) \nabla_{\perp} \phi\left(\mathbf{r}_{\perp}, z^{\prime}\right)\right\}=-\left.k_{\mathrm{av}} \frac{\partial I\left(\mathbf{r}_{\perp}, z\right)}{\partial z}\right|_{z=z^{\prime}},
$$

where the averages are defined for polychromatic wave fields with mean wave number $k_{\text {av }}$ (Ref. 5) and $\mathbf{r}_{\perp}$ is a Cartesian vector orthogonal to the $z$-axis. A practical solution of Eq. (1) for $\phi\left(\mathbf{r}_{\perp}, z\right)$ was developed by Paganin and Nugent. ${ }^{5}$ The TIE phase retrieval approach has since been used in TEM to map phase shifts induced by magnetic and electrostatic potentials from nanoparticles. ${ }^{6-9}$ We have recently shown ${ }^{10,11}$ that estimation of the TIE $z$-derivative using polynomial fitting for the $z$-variation in $I\left(\mathbf{r}_{\perp}, z^{\prime}\right)$ yields robust measurement of $\phi\left(\mathbf{r}_{\perp}, z^{\prime}\right)$ on the nanoscale.

In this work we report quantitative TIE electron phase maps from Au and polystyrene (PS) nanospheres in conjunction with EELS surface plasmon maps. Some theoretical analysis is also presented, which was conducted to explore the origin of electron phase shifts detected near Au nanospheres.

PS spheres with sizes of 30-100 nm in diameter (manufactured by JSR Corporation) were combined with $\mathrm{Au}$

${ }^{a)}$ Electronic mail: tim.petersen@emu.usyd.edu.au. spheres, nominally $25 \mathrm{~nm}$ in diameter (manufactured by Aurion) in a dilute ethanol solution, agitated in an ultrasonic bath, then dispersed onto a carbon film. STEM measurements of adjacent PS and Au spheres were performed using a VG HB601UX microscope equipped with a cold field emission gun (CFEG), operated at $100 \mathrm{kV}$. The CFEG extraction voltage was lowered to reduce the chromatic spread of electrons emitted from the source, ${ }^{12}$ resulting in a diminished beam current. To compensate, a relatively large STEM probe of about $3 \mathrm{~nm}$ was used, producing coarser spatial sampling, yet deemed sufficient in consideration of delocalisation effects. $^{13,14}$ The convergence angle employed was $11 \mathrm{mrad}$.

Twelve through-focus bright field images were collected on the VG STEM, using small increments of the objective lens strength. In-focus and defocus derivative maps were calculated by fitting a Taylor series to the focus variations at each point in every image, assuming the objective lens current to be proportional to the defocus, which was not calibrated. The fitting was performed using a robust algorithm based on singular value decomposition, adapted from Ref. 15. Qualitative phase information was then retrieved using the Paganin-Nugent approach. ${ }^{5}$ A small degree of high-pass filtering was used to remove excessive low-spatial frequency contributions from the resulting phase map.

EELS data were collected on the VG STEM with a Gatan Enfina EELS detector, using the method of binned gain averaging. ${ }^{16}$ The effective acquisition time per spectrum was $4.3 \mathrm{~ms}$, allowing good sampling over the otherwise detrimental background 50 and $150 \mathrm{~Hz}$ main oscillations. ${ }^{17}$ The collection angle used was $3 \mathrm{mrad}$. After postacquisition EELS alignment and subtraction of dark current, zero-loss peak contributions were removed from all spectra by subtracting a high-quality vacuum spectrum, fitted between 0.8 and $1.2 \mathrm{eV}$. A plasmon map was then calculated by integrating the spectral intensity between 2.35 and $2.45 \mathrm{eV}$.

Figure 1 compares the EELS and TIE phase maps for the adjacent $\mathrm{Au}$ and PS spheres, showing some similarities. In the absence of external fields and diffraction effects, the TIE 


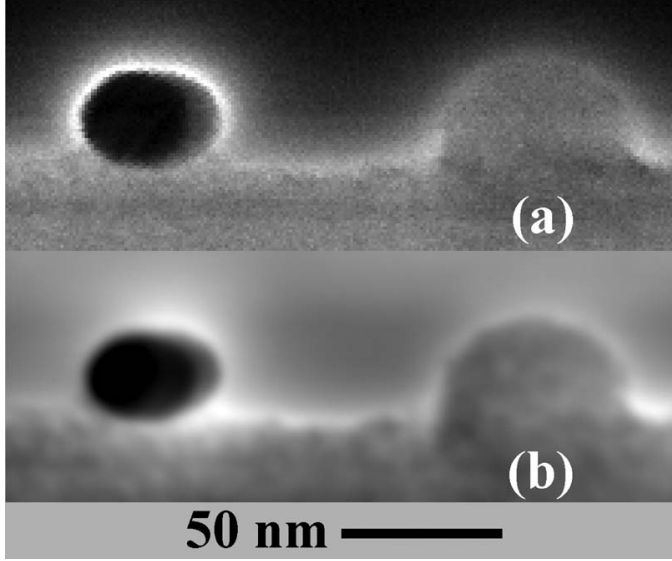

FIG. 1. (a) EELS surface plasmon map for Au and PS spheres. (b) TIE phase map from the same specimen area.

phase for elastic scattering should approximate a thickness map for the Au and PS spheres, with no phase gradients in the vacuum. The phase excursions near the Au sphere in the TIE phase map are therefore unexpected. Quantification of the phase in Fig. 1(b) is difficult since the results were sensitive to image alignment due to noise in the STEM data. The possibility of nonphysical artifacts in the phase data cannot be ruled out. We therefore carried out additional experiments on a $300 \mathrm{kV}$ JEOL 3000F TEM to improve data quality for quantitative phase retrieval.

Using a small condenser aperture, the $300 \mathrm{kV}$ TEM was aligned to produce a parallel beam of roughly $500 \mathrm{~nm}$ in diameter and a Gatan imaging filter was employed to remove inelastic scattering contributions, with a $10 \mathrm{eV}$ slit width centered about the zero-loss peak. A modulation transfer function (MTF) for the charge coupled device detection system was measured using the "noise method" 18 as described elsewhere. ${ }^{11}$

For TIE phase benchmarking purposes, an isolated $100 \mathrm{~nm}$ diameter PS sphere was chosen for analysis. Twenty-five through-focus images were collected, spanning a total calibrated defocus range from $z=-4800 \mathrm{~nm}$ to $z=+4800 \mathrm{~nm}$. The particular sphere chosen appeared less susceptible to beam degradation than other spheres that were examined. All images were carefully aligned and padded to their original boundaries with intensity averages. The effects of the MTF were deconvolved from the zeroth and first order defocus derivatives using an iterative nonlinear algorithm known as "algebraic update," adapted from Jansson. ${ }^{19}$ The Paganin-Nugent ${ }^{5}$ algorithm was then used to invert the TIE for the phase, without any bandpass filtering, producing the map shown in Fig. 2(a).
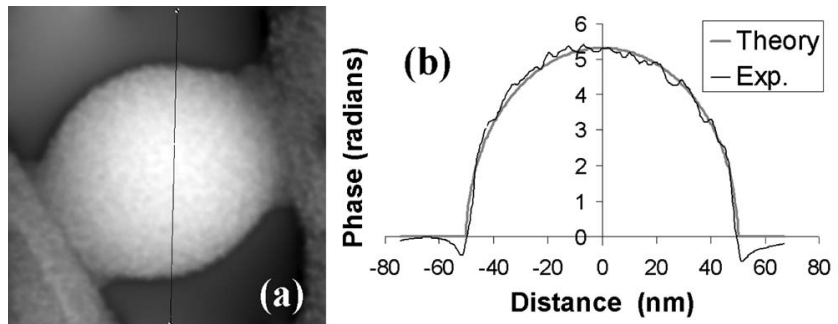

FIG. 2. (a) Quantitative TIE phase map for a $100 \mathrm{~nm}$ PS sphere (contrast inverted). The gray scale varies linearly from -2.8 to 5.6 rad. (b) Quantitative TIE phase profile for the $100 \mathrm{~nm}$ PS sphere.

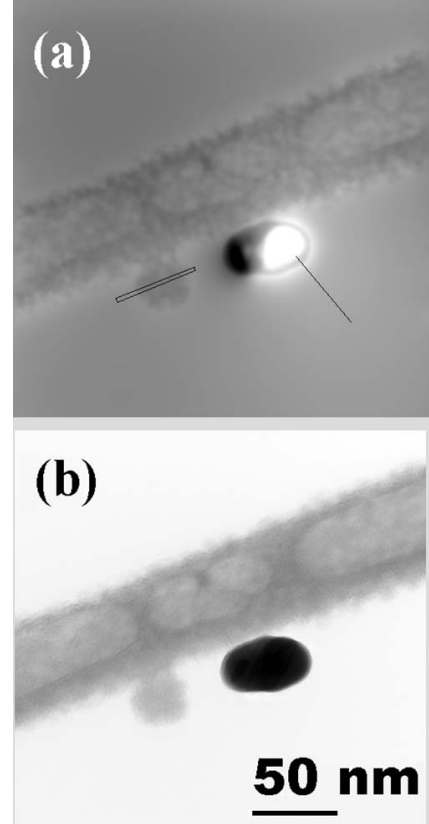

FIG. 3. (a) TIE phase map; the gray scale contrast varies linearly from -12.5 to $7.5 \mathrm{rad}$. (b) In-focus image.

For a weakly scattering homogenous sphere in the absence of external fields, the phase shift relative to the vacuum $\phi\left(\mathbf{r}_{\perp}\right)$ can be approximated as $\phi\left(\mathbf{r}_{\perp}\right)=-\sigma T\left(\mathbf{r}_{\perp}\right) V_{0},{ }^{20}$ where $V_{0}$ is the "mean inner potential," $T\left(\mathbf{r}_{\perp}\right)$ is the thickness of the specimen, and $\sigma$ is an interaction constant determined by the TEM accelerating voltage. $V_{0}$ is a specimendependent quantity, which can be measured using a variety of methods. Figure 2(b) shows the (inverted) phase shift along the line drawn in Fig. 2(a), which was fitted to a spherical profile to give an implied mean inner potential of about $V_{0}=8.1 \mathrm{~V}$. This value is in accordance with PS sphere off-axis electron holography measurements in the literature, ${ }^{21}$ which reported $V_{0}$ as $8.5 \pm 0.7 \mathrm{~V}$.

Phase measurements were performed for a second region of the specimen, containing adjacent Au and PS spheres, using 25 images between $z=-1800 \mathrm{~nm}$ and $z=+1800 \mathrm{~nm}$ and slight high-pass filtering to produce the data shown in Fig. 3(a). The leftmost line drawn in Fig. 3(a) indicates a region used to measure the (inverted) quantitative phase profile shown in Fig. 4(a), which was calculated using the $V_{0}$ value from Fig. 2(b) and a measured diameter of $32 \mathrm{~nm}$. Figure 4(b) shows a similar profile taken from just inside the edge of the Au sphere toward the bottom right corner of Fig. 3(a). Figure 4(a) is consistent with prior observations from Fig. 1. In contrast, Fig. 4(b) shows large phase excursions near the Au sphere that decay into the vacuum; the sphere edge coincides with the second maximum. The PS sphere phase appears devoid of such effects. Figure 3(a) shows mottled contrast for the PS sphere and carbon support, which is due to electron beam damage. However, the theoretically consistent absolute scale and shape of the profile in Fig. 4(a) suggest an accurate retrieval of the TIE phase in this image. These results confirm the presence of large phase shifts near the edge of metal nanoparticles, which are not observed near insulating nanoparticles, as also shown in Fig. 1.

Assuming dipole oscillations of the $\mathrm{Au}$ sphere charge distribution and excluding retardation effects, the phase pro- 

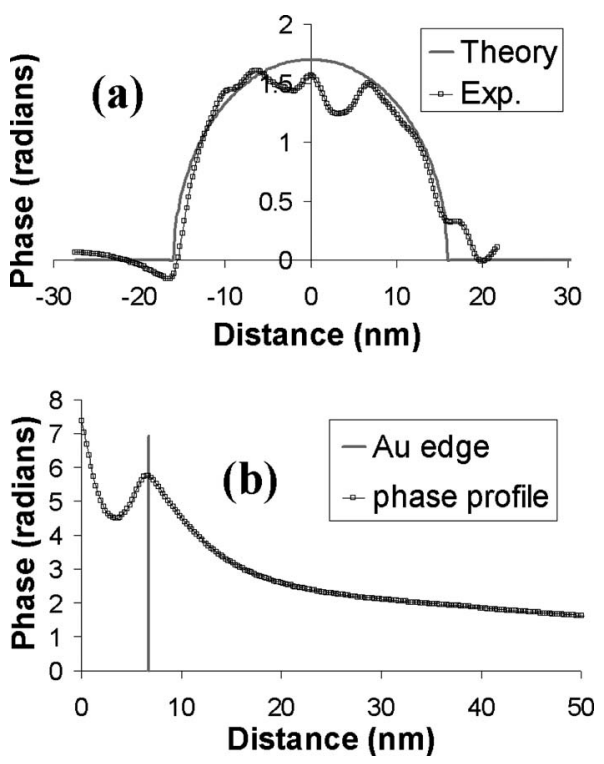

FIG. 4. (a) Inverted phase profile along the PS sphere shown in Fig. 3(a). (b) Phase profile for the vacuum near the Au sphere (the peak around $7 \mathrm{~nm}$ corresponds to the edge of the Au particle).

file expected from elastic interactions can be derived using an image-potential approach. Disregarding dynamical scattering contributions, the accumulated phase shift induced upon the TEM electron with speed $v$ by the Au sphere with dielectric function $\varepsilon(\omega)$ is then

$$
\begin{aligned}
\phi \cong & \frac{e}{\hbar v} \frac{2 a^{3}}{\pi^{2}} \frac{e}{v^{3}} \int \mathfrak{R}\left[\frac{\varepsilon(\omega)-1}{\varepsilon(\omega)+2}\right] \\
& \times \omega^{2}\left\{K_{1}\left(\frac{\omega R}{v}\right)^{2}+K_{0}\left(\frac{\omega R}{v}\right)^{2}\right\} d \omega,
\end{aligned}
$$

where $a$ is the sphere radius, $R$ is the radial distance from the center of the sphere to the point on the plane at which the phase is measured, and $K_{0}$ and $K_{1}$ are modified Bessel functions of the second kind. Note that $\mathfrak{R}$ denotes the real part of the sphere's polarizability; for measures of inelastic absorption, the imaginary part is taken. ${ }^{22}$ Calculations using Eq. (2) yield a phase profile similar to Fig. 3(b), yet several orders of magnitude smaller. Further, we have generalized Eq. (2) to include multipole oscillations and found only marginal increase in the expected phase shift. Similar considerations of induced magnetic effects lead to the same conclusion. Our calculations suggest that the derived phase profile should nonetheless be measurable using off-axis electron holography, as the recent literature has shown that milli- radian sensitivity can be achieved for resolutions of about $5-10 \mathrm{~nm}^{23}$

The failure of elastic interactions to explain the observed phase excursions suggests that inelastic scattering must be producing the observed phase changes. The incoherence between electrons of different energies will mean that the final observed phase shift will simply be a sum of the phase shifts from each inelastic contribution. Indeed, Eq. (1) holds for polychromatic wave fields. ${ }^{5}$

In conclusion, we have demonstrated a phase-based method for visualizing surface plasmon excitation in metal nanostructures, which may be of considerable practical benefit for research in the field of plasmonics. In future work we plan to use energy-filtered TEM with higher energy resolution to remove surface plasmon contributions from images in the TIE and also pursue quantitative predictions using inelastic scattering theory.

${ }^{1}$ P. E. Batson, Phys. Rev. Lett. 49, 936 (1982).

${ }^{2}$ M. Bosman, V. J. Keast, M. Watanabe, A. I. Maaroof, and M. B. Cortie, Nanotechnology 18, 165505 (2007).

${ }^{3}$ H. Lichte, Philos. Trans. R. Soc. London, Ser. A 360, 897 (2002).

${ }^{4}$ M. R. Teague, J. Opt. Soc. Am. 73, 1434 (1983).

${ }^{5}$ D. Paganin and K. A. Nugent, Phys. Rev. Lett. 80, 2586 (1998).

${ }^{6}$ M. Beleggia, M. A. Schofield, V. V. Volkov, and Y. Zhu, Ultramicroscopy 102, 37 (2004).

${ }^{7}$ S. Bajt, A. Barty, K. A. Nugent, M. McCartney, M. Wall, and D. Paganin, Ultramicroscopy 83, 67 (2000).

${ }^{8}$ V. V. Volkov and Y. Zhu, Phys. Rev. Lett. 91, 043904 (2003).

${ }^{9}$ A. Kohn, A. K. Petford-Long, and T. C. Anthony, Phys. Rev. B 72, 014444 (2005).

${ }^{10}$ T. Petersen, V. Keast, K. Johnson, and S. Duvall, Philos. Mag. 87, 3565 (2007).

${ }^{11}$ T. C. Petersen, V. J. Keast, and D. M. Paganin, Ultramicroscopy 108, 805 (2008).

${ }^{12}$ A. V. Crewe, M. Isaacson, and D. Johnson, Rev. Sci. Instrum. 42, 411 (1971).

${ }^{13}$ R. F. Egerton, Ultramicroscopy 107, 575 (2007).

${ }^{14}$ D. A. Muller and J. Silcox, Ultramicroscopy 59, 195 (1995).

${ }^{15}$ W. H. Press, B. P. Flannery, S. A. Teukolsky, and B. P. Flannery, Numerical Recipes in C: The Art of Scientific Computing (Cambridge University Press, Cambridge, 1992).

${ }^{16}$ M. Bosman and V. J. Keast, Ultramicroscopy 108, 837 (2008).

${ }^{17}$ G. Y. Fan and M. H. Ellisman, J. Microsc. 200, 1 (2000).

${ }^{18}$ J. M. Zuo, Ultramicroscopy 66, 21 (1996).

${ }^{19}$ Deconvolution of Images and Spectra, 2nd ed., edited by P. A. Jansson (Academic, San Diego, 1997).

${ }^{20}$ Electron Diffraction Techniques, edited by J. M. Cowley (Oxford University Press, Oxford, 1992), Vol. 1.

${ }^{21}$ Y. C. Wang, T. M. Chou, M. Libera, E. Voelkl, and B. G. Frost, Microsc. Microanal. 4, 146 (1998).

${ }^{22}$ H. Kohl, Ultramicroscopy 11, 53 (1983).

${ }^{23}$ D. Cooper, R. Truche, P. Rivallin, J.-M. Hartmann, F. Laugier, F. Bertin, and A. Chabli, Appl. Phys. Lett. 91, 143501 (2007). 